\title{
ON COUPLED OSCILLATORS NETWORKS \\ - FOR THE CELLULAR NEURAL NETWORK -
}

\author{
Yoshifumi NISHIO $\dagger$, Shinsaku MORI †and Akio USHIDA $\ddagger$ \\ $\dagger$ Dept. of Electrical Engineering, Keio University \\ 3-14-1 Hiyoshi, Kouhoku-ku, Yokohama, 223 Japan \\ Tel. +81-45-563-1141 Ext. 3319 \\ Fax. $+81-45-563-2773$ \\ Email nishio@mori.elec.keio.ac.jp \\ $\ddagger$ Dept. of Electrical Engineering, Tokushima University \\ 2-1 Minami-Jousanjima, Tokushima, 770 Japan
}

\begin{abstract}
In this study, we propose two types of coupled oscillators networks and investigate their steady states. One network has two-dimensional honeycomb structure. The other network has two-dimensional lattice structure. In the Honeycomb circuit, adjacent three oscillators are coupled by one coupling resistor and it is considered that the voltages of three oscillators around a coupling resistor make three-phase synchronization. On the other hand, in the Lattice circuit, four-oscillators are coupled by one coupling resistor and it is considered that the voltages of four oscillators around a coupling resistor make four-phase synchronization. As a result, our networks are expected to generate various synchronization patterns. In our networks each oscillator is connected to only its adjacent oscillators and various patterns are generated according to the initial condition. Therefore, our network is expected to be utilized as a cellular neural network in the future.
\end{abstract}

\section{INTRODUCTION}

Cellular neural network proposed by Chua and Yang [1] have two major features; continuous time feature allowing real-time signal processing and local interconnection feature making it tailor made for VLSI implementation. And various applications for image processing and pattern recognition of cellular neural network have been reported.

Recently, neural network using a van der Pol oscillator as a neuron has been proposed by Endo et al [2]. This system has the advantage that no annealing is needed to achieve good convergence to an optimum solution. Because the static characteristic of the van der Pol oscillator is completely binary and the dynamic characteristic is continuous and smooth. Moreover, it achieve fast convergence. Therefore, coupled oscillators systems are expected to be utilized for the neural network.

On the other hand, there have been many investigations of the mutual synchronization of oscillators ([3]-[5] and therein). We have reported synchronization phenomena observed from 0-7803-1254-6/93\$03.00 @ 1993 IEEE
$N$ oscillators with the same natural frequency mutually coupled by one resistor [5]. The system is shown in Fig. 1. In the system various synchronization phenomena can be stably observed, because the system tends to minimize the current through the coupling resistor. Especially, we have confirmed experimentally that $N$-phase oscillation ( $N=2 \sim 13)$ can be stably excited for the case that each oscillator has strong nonlinearity. In this case, there exist $(N-1)$ ! phase states. Because of the coupling structure and extremely large number of phase states of the system in Fig. 1, this system would be structural element of the cellular neural network.
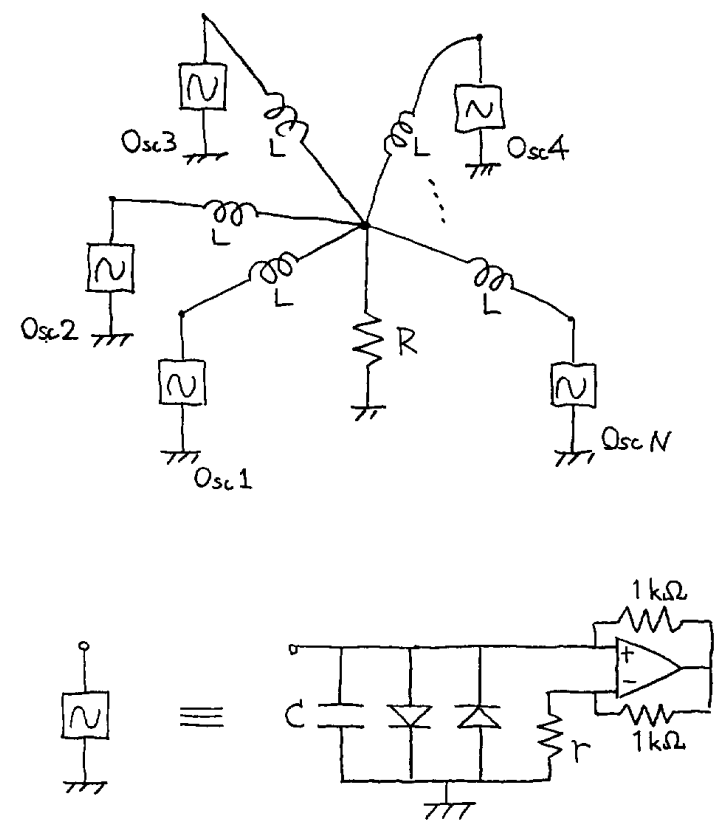

Fig. 1 Basic circuit model used as a structural element of the network. 


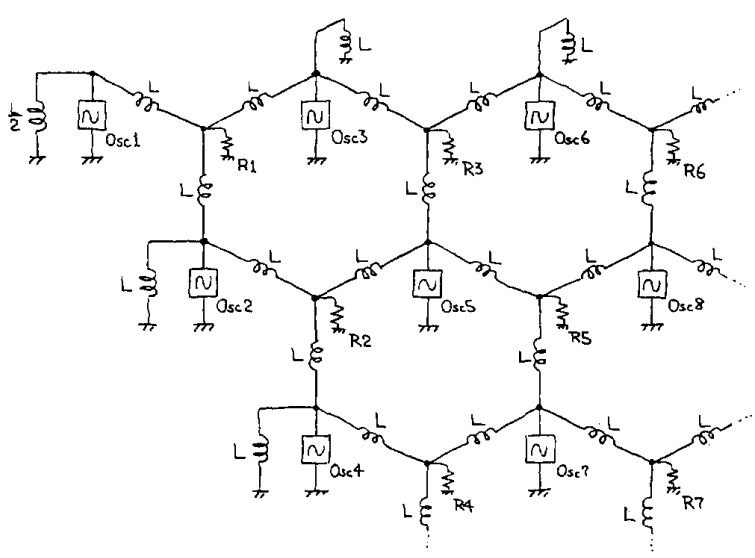

Fig. 2 Coupled oscillators network (Honeycomb circuit).

In this study, we propose two types of coupled oscillators networks whose structural element is the circuit in Fig. 1 and investigate their steady states. One network has twodimensional honeycomb structure and it is shown in Fig. 2. Also the other network has two-dimensional lattice structure and it is shown in Fig. 3. In the Honeycomb circuit, three oscillators are coupled by one coupling resistor and it is considered that the voltages of three oscillators around a coupling resistor make three-phase synchronization according to the result in [3]. On the other hand, in the Lattice circuit, four oscillators are coupled by one coupling resistor and it is considered that the voltages of four oscillators around a coupling resistor make four-phase synchronization. Therefore, our networks can generate various synchronization patterns. We carry out circuit experiments for two sizes of Honeycomb circuit and one size of Lattice circuit and confirm the generation of various patterns.

In our networks each oscillator is connected to only its adjacent oscillators and various patterns are generated according to the initial condition. Therefore, our network is expected to be utilized as a cellular neural network in the future.

\section{HONEYCOMB CIRCUIT}

Fig. 2 shows the Honeycomb circuit. In this circuit adjacent three oscillators (e.g. Osc1, Osc2 and Osc3 in Fig. 2) are coupled by one coupling resistor (R1 in Fig. 2). Therefore, it is considered that the voltages of these three oscillators make three-phase synchronization according to the result in [3]. In the Honeycomb circuit, only 2 steady states can be exist in spite of the size of the network. Because if the relation of the phases of three oscillators around a coupling resistor is decided, the phase of the next oscillator is decided uniquely. This is confirmed by circuit experiment and computer calculation for two sizes of the network; one has equilateral triangle form with three oscillators on one side (see Fig. 4(a)) and the

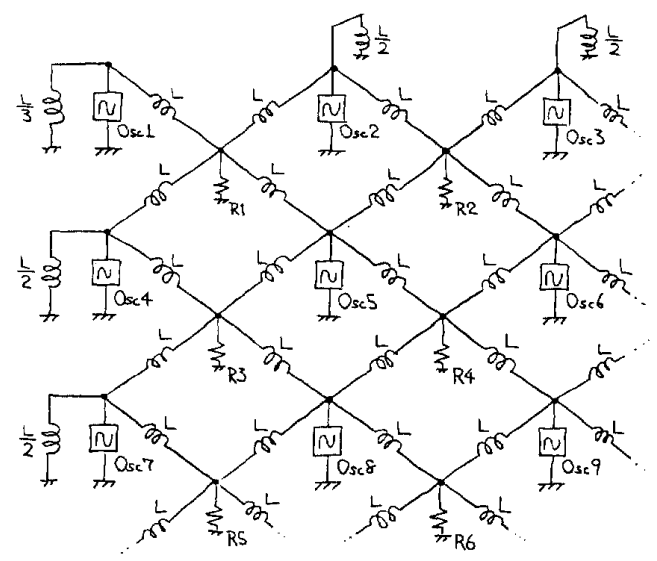

Fig. 3 Coupled oscillators network (Lattice circuit).

other has equilateral triangle form with four oscillators on one side (see Fig. 5(a)). We call the former as Honeycomb-1 and the latter as Honeycomb-2. The circuit experimental results are shown in Figs. 4 and 5 . In both figures, $v_{k}$ is the voltage of the Osck and the notations $A, B$ and $C$ are mean the three phase states. Though we omit the computer calculation results, two synchronization patterns corresponding to Figs. 4(b)(c) and 5(b)(c) can be obtained.

In this circuit model, there exist only two patterns. However, it is considererd that various synchronization can be stably excited by inputting some signals. The investigation of the generation of such new patterns is our future problem.

\section{LATTICE CIRCUIT}

Fig. 3 shows the Lattice circuit, In this circuit adjacent four oscillators (e.g. Osc1, Osc2, Osc4, and Osc5 in Fig. 3) are coupled by one coupling resistor (R1 in Fig. 3) Therefore, it is considered that the voltage of these four oscillators make four-phase synchronization. In this circuit, it is proved that there exist $3 !\left(2^{N_{1}-3}+2^{N_{2}-3}-1\right)$ patterns $\left(N_{1} \times N_{2}\right.$ is the size of the network). Two typical examples of such patterns for the size $3 \times 3$ obtained from circuit experiments are shown in Fig. 6. In Fig. 6 the notations $A \sim D$ mean four phase states.

Although we have not analyzed the control of the output patterns by external inputs, we consider that several patterns can be obtained by inputting some signals to some oscillators. Therefore, we believe that we can control the output patterns by external inputs and that some applications using our network as a cellular neural network will be proposed in near future.

\section{CONCLUSIONS}

In this study, we have proposed two types of coupled oscillators networks and have investigated their steady states. One 

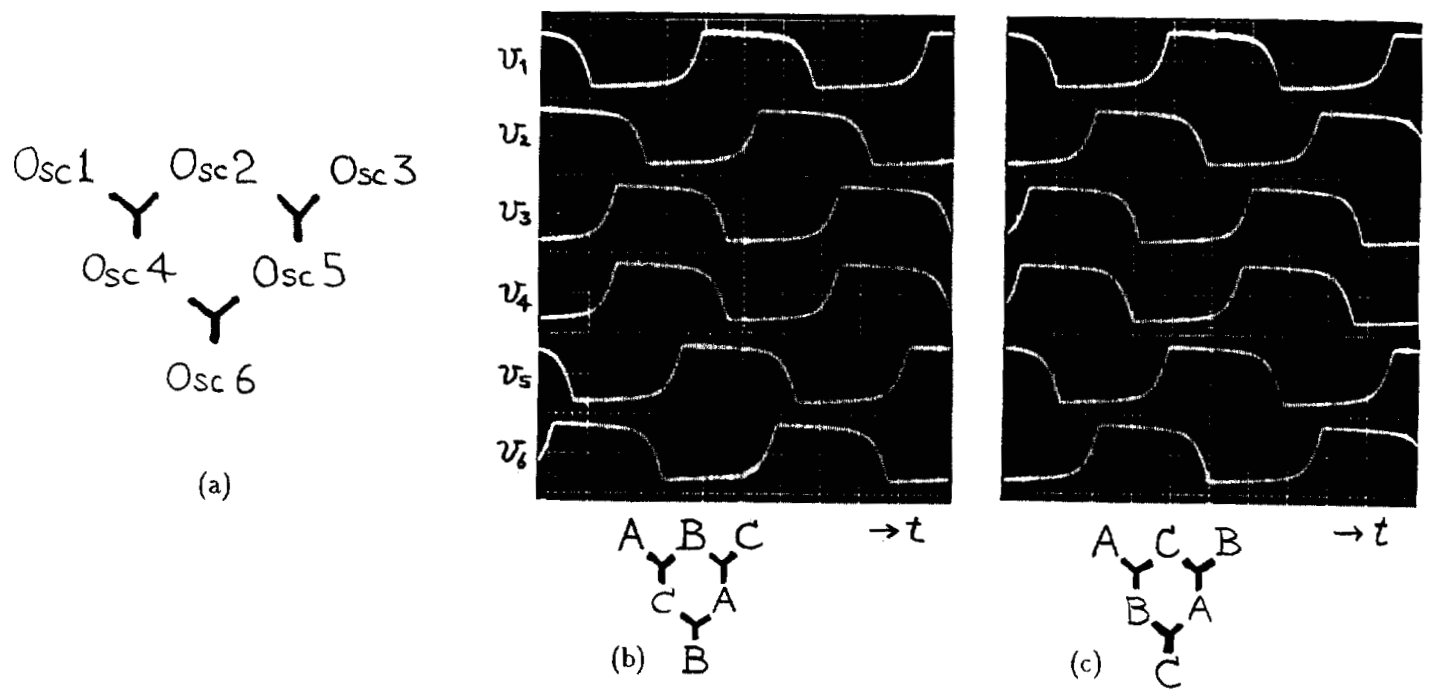

Fig. 4 Circuit experimental results for the Honeycomb-1 $(L=9.9 \mathrm{mH}, \quad C=0.01 \mu F, \quad R=1.5 k \Omega, \quad r=300 \Omega)$. (horizontal axis : $10 \mu \mathrm{sec} /$ div., vertical axis : $1 \mathrm{~V} /$ div.).

(a) Arrangement of the oscillators. (b) Pattern 1. (c) Pattern 2.
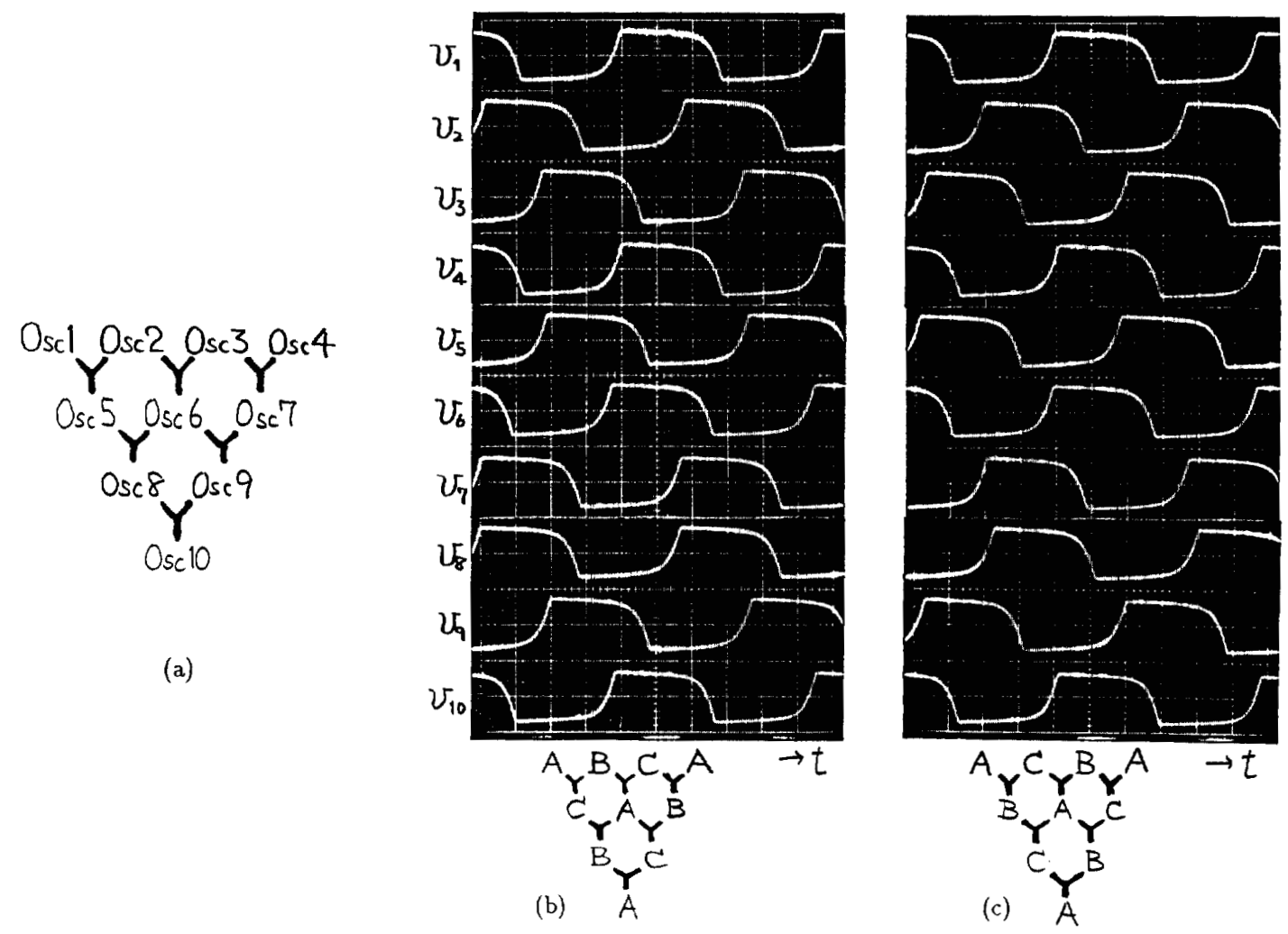

Fig. 5 Circuit experimental results for the Honeycomb-2 (Parameter values are the same as those in Fig. 4) (horizontal axis : $10 \mu \mathrm{sec} /$ div., vertical axis : $1 \mathrm{~V} /$ div.).

(a) Arrangement of the oscillators. (b) Pattern 1. (c) Pattern 2. 


\section{${ }^{0 s 4} x^{0 s c 5} x^{0 s c 6}$ \\ Osc7 0 Osc 8 Osc9}

(a)
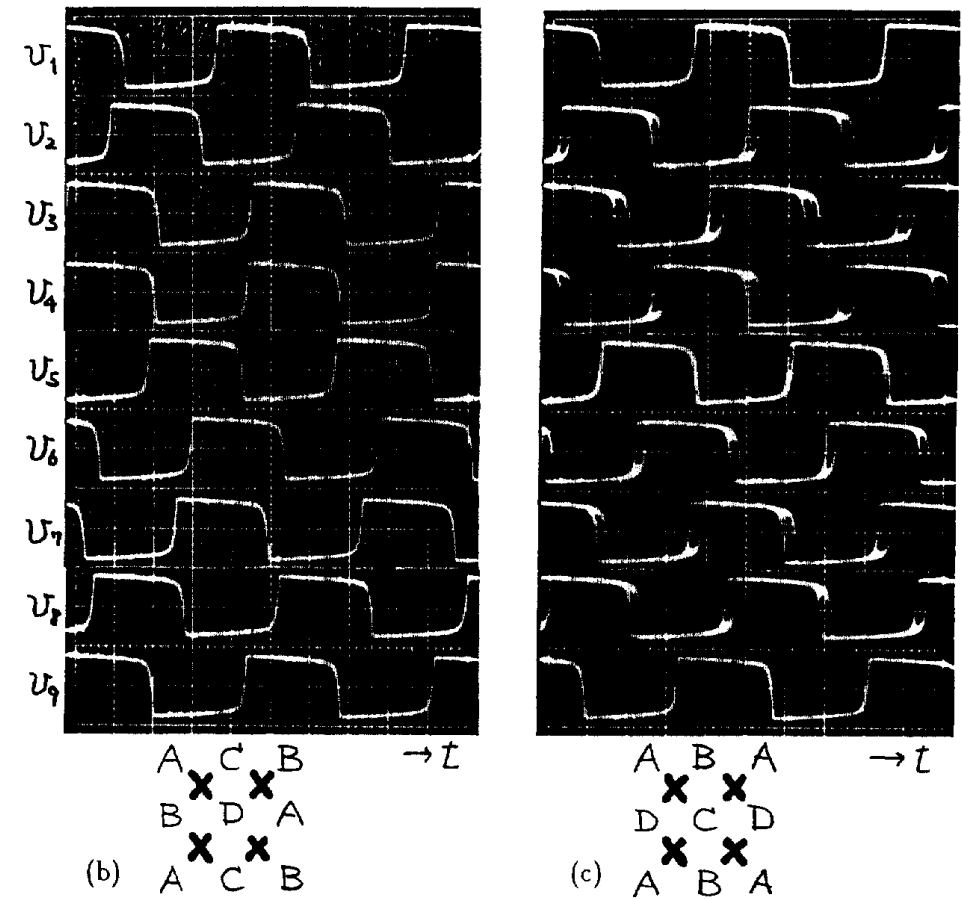

Fig. 6 Circuit experimental results for the Lattice circuit $(L=9.9 \mathrm{mH}, \quad C=0.01 \mu F, \quad R=1.5 \mathrm{k} \Omega, \quad r=100 \Omega)$. (horizontal axis : $20 \mu \mathrm{sec} /$ div., vertical axis : $1 \mathrm{~V} /$ div.).

(a) Arrangement of the oscillators. (b) Pattern 1. (c) Pattern 2.

network has two-dimensional honeycomb structure. Also the other network has two-dimensional lattice structure. In our networks each oscillator is connected to only its adjacent oscillators and various patterns are generated according to the initial condition. Therefore, our network is expected to be utilized as a cellular neural network in the future.

\section{REFERENCES}

[1] L. O. Chua and L. Yang, "Cellular Neural Networks: Theory," IEEE Trans. Circuits Syst., Vol. CAS-35, No. 10 , (1988), 1257.

[2] T. Endo, L. O. Chua and K. Takeyama, "A Neural Network Using Oscillators," Proc. of ISCAS'91, (1991), 782.

[3] H. Kimura and K. Mano, "Some Properties of Mutually Synchronized Oscillators Coupled by Resistances," Trans. IECE, Vol. 48, No. 10, (1965), 1647(in Japanese).

[4] T. Endo and S. Mori, "Mode Analysis of a Ring of a Large Number of Mutually Coupled van der Pol Oscillators," IEEE Trans. Circuits Syst., Vol. CAS-25, No. 1, (1978), 7.
[5] Y. Nishio and S. Mori, "Mutually Coupled Oscillators with an Extremely Large Number of Steady States," Proc. of ISCAS'92, (1992), 819. 